\title{
Chemotaxis of a Ralstonia sp. SJ98 toward co-metabolizable nitroaromatic compounds
}

\author{
Gunjan Pandey, Ashvini Chauhan, ${ }^{1}$ Sudip K. Samanta, ${ }^{2}$ and Rakesh K. Jain* \\ Institute of Microbial Technology, Sector 39-A, Chandigarh 160036, India
}

Received 10 October 2002

\begin{abstract}
We have earlier reported chemotaxis of a Gram-negative, motile Ralstonia sp. SJ98 towards $p$-nitrophenol (PNP), 4-nitrocatechol (NC), $o$-nitrobenzoate (ONB), $p$-nitrobenzoate (PNB), and 3-methyl-4-nitrophenol (MNP) that also served as sole source of carbon and energy to the strain [S.K. Samanta, B. Bhushan, A. Chauhan, R.K. Jain, Biochem. Biophy. Res. Commun. 269 (2000) 117; B. Bhushan, S.K. Samanta, A. Chauhan, A.K. Chakraborti, R.K. Jain, Biochem. Biophy. Res. Commun. 275 (2000) 129]. In this paper, we report chemotaxis of a Ralstonia sp. SJ98 toward seven different nitroaromatic compounds (NACs) by drop assay, swarm plate assay, and capillary assay. These NACs do not serve as sole carbon and energy source to strain SJ98 but are partially transformed in the presence of an alternate carbon source such as succinate. This is the first report showing chemotaxis of a bacterial strain toward co-metabolizable NACs.
\end{abstract}

(c) 2002 Elsevier Science (USA). All rights reserved.

Keywords: Chemotaxis; Co-metabolism; Nitroaromatic compounds; Bioremediation; Ralstonia sp.

The use of microbial metabolic potential for elimination of pollution is now accepted as a safe and economic alternative to other physico-chemical methods that are less efficient and cost-ineffective [1-4]. Bacterial chemotaxis, movement under influence of a chemical gradient, is reasonably argued to enhance biodegradation as it increases bioavailability of a pollutant to the bacteria. However, this aspect of bioremediation has received less attention, although some microorganisms with chemotactic ability toward different xenobiotic compounds have been isolated and characterized [5-10].

NACs are widely present in the environment because of their extensive use in the production of insecticides, pesticides, plastics, dyes, and explosives [11]. The stability, persistence, and toxicity make NACs hazardous when released into the environment. Due to their toxic, mutagenic, and carcinogenic nature [11], a number of NACs

\footnotetext{
${ }^{*}$ Corresponding author. Fax: +91-172-690-585/690-632.

E-mail address: rkj@imtech.res.in (R.K. Jain).

${ }^{1}$ Present address: Soil and Water Science, 2169, Mercarty Hall, University of Florida, FL 32611, USA.

${ }^{2}$ Present address: Department of Microbiology, University of Iowa, 3-401 Bowen Science Building, Iowa City, IA 52242, USA.
}

including nitrobenzenes, nitrotoluenes, and nitrophenols are listed as priority pollutants by "United States Environmental Protection Agency" [12]. In the past few decades, a number of microorganisms have been isolated and characterized which are capable of degrading a wide range of NACs $[11,13]$. In most instances where mineralization has been demonstrated, the catabolic pathway and its regulation have also been determined.

A Ralstonia sp. SJ98 has been shown to be chemotactic toward PNP, NC, ONB, PNB, and MNP [5,6]; these compounds also served as source of carbon and energy to the organism. In this paper, we report chemotaxis of strain SJ98 toward seven other nitroaromatic compounds that do not serve as sole source of carbon and energy to the organism but are co-metabolized.

\section{Materials and methods}

\footnotetext{
Microorganisms and culture conditions. A Ralstonia sp. SJ98 was isolated in our laboratory by "chemotactic enrichment technique" from a pesticide-contaminated soil sample [5]. The composition of minimal medium (MM) used in the present study was the same as that described earlier [14]. Different NACs (o-dinitrobenzene, $m$-dinitrobenzene, $o$-nitrophenol, $m$-nitrophenol, 2,4-dinitrophenol, 2,5-dinitrophenol, 2,6-di-
} 
nitrophenol, $p$-nitroaniline, 2,3-dinitrotoluene, and 3,5-dinitrobenzoic acid) were added as filter sterilized solutions into MM at a final concentration of $0.3 \mathrm{mM}$ each. Filter sterilized $10 \mathrm{mM}$ succinate that served as an alternate source of carbon and energy to the cells was also added in the MM. The medium was inoculated with overnight grown seed culture and incubated at $30^{\circ} \mathrm{C}$ under shaking conditions (200 rpm). The presence of nitrite and ammonia in the culture supernatant was determined by previously described methods [5].

Extraction of intermediates and analytical methods. To study the transformation products of different NACs by strain SJ98 extraction was carried out following overnight growth of strain SJ98 as described above. The culture supernatants were extracted with an equal volume of ethyl acetate (neutral extraction) and then the $\mathrm{pH}$ of the aqueous phase was adjusted to 2.0 with $5 \mathrm{M} \mathrm{HCl}$ and again extracted with equal volume of ethyl acetate (acidic extraction) [5]. The neutral and acidic extracts were mixed and evaporated to dryness using a rotary evaporator, dissolved in $100 \mu \mathrm{l}$ methanol, and analyzed for transformation products by thin layer chromatography (TLC) and gas chromatography (GC) as described earlier [15]. Appropriate controls, as required, were also used in these experiments.

High performance liquid chromatography (HPLC) using a Waters 600 model was also carried out to study the transformation of different NACs by strain SJ98 following growth as described above. Detection was done by scanning the samples at $190-300 \mathrm{~nm}$ with a Waters 996 photodiode array detector. Separation was carried out with a Waters Spherisorb $5 \mu \mathrm{m} \mathrm{C8}$ column and the separating conditions were as follows: temperature $25^{\circ} \mathrm{C}$; mobile phase $50 \%$ aqueous methanol ( $0.1 \%$ trifluoroacetic acid); and isocratic flow rate, $1.5 \mathrm{ml} / \mathrm{min}$.

Chemotaxis assays. The chemotactic behavior of strain SJ98 was studied by three established methods, viz., drop assay, swarm plate assay, and capillary assay, which have been standardized in our laboratory [5-7].

For drop assay, cells were grown in nutrient broth and induced at early log phase $\left(\mathrm{OD}_{600} 0.3\right)$ with $0.2 \mathrm{mM}$ of the nitroaromatic compound (NAC) in question. They were grown further for $3-5 \mathrm{~h}$, washed and resuspended in drop assay medium (MM containing $0.30 \%$ bacto agar) and poured in petri plates. Crystals of NAC were placed in the center of the petri plate and chemotactic response of Ralstonia sp. SJ98 was observed within $3-4 \mathrm{~h}$ of incubation at room temperature $\left(25^{\circ} \mathrm{C}\right)$.

For swarm plate assay, the NAC (final concentration $0.2 \mathrm{mM}$ ) was added to the swarm plate medium (MM containing $0.16 \%$ bacto agar) before pouring the plates. About $75-100 \mu \mathrm{l}$ of induced and washed cell suspension $\left(\mathrm{OD}_{600} 2.0\right)$ in $\mathrm{MM}$ was gently poured at the center of the plate and incubated at room temperature $\left(25^{\circ} \mathrm{C}\right)$. Here, $1 \mathrm{mM}$ glucose was added to the cell suspension for providing energy to the cells. Formation of rings was observed after $12-16 \mathrm{~h}$ of incubation.

For capillary assays, capillary tubes (Drummond Scientific, USA) of $1 \mu \mathrm{l}$ capacity were used. The chemotaxis buffer consisted of $100 \mathrm{mM}$ potassium phosphate buffer (pH 7.2) and $20 \mu \mathrm{M}$ EDTA. The appropriate NAC was added to the buffer in the final concentrations of 10 , 20 , and $200 \mu \mathrm{M}$. Aspartic acid $(200 \mu \mathrm{M})$ was used as positive control.
These solutions were filled into capillaries that were then inserted into the cell suspensions $\left(10^{6}-10^{7}\right.$ cells $/ \mathrm{ml}$ chemotaxis buffer $)$ on a glass slide. After incubation for $30 \mathrm{~min}$, cells from the capillary were serially diluted and plated onto nutrient agar. Colonies were counted after overnight growth at $30^{\circ} \mathrm{C}$. The capillary tube containing buffer alone was used as control. The chemotaxis index (C.I.) was determined as the ratio of the number of bacterial cells accumulated in the test capillary containing NAC to that in control.

Chemicals. All the NACs used were purchased from Sigma or Aldrich, USA. Analytical and spectroscopic grade ethyl acetate and methanol were purchased from E. Merck, Germany. All other chemicals were of highest purity grade commercially available.

\section{Results and discussion}

Strain SJ98, which is chemotactic toward PNP, NC, $\mathrm{ONB}, \mathrm{PNB}$, and MNP that also serve as sole source of carbon and energy to the strain [5,6], was tested for its ability to transform a wide range of NACs in this study. These compounds that do not serve as sole source of carbon and energy to strain SJ98 included $o$-dinitrobenzene, $m$-dinitrobenzene, $o$-nitrophenol, $m$-nitrophenol, 2,4-dinitrophenol, 2,5-dinitrophenol, 2,6-dinitrophenol, $m$-nitroaniline, 2,3-dinitrotoluene, and 3,5-dinitrobenzoic acid. To determine whether these NACs are transformed, oxidatively or reductively, strain SJ98 was grown on MM [14] containing $10 \mathrm{mM}$ succinate and $0.3 \mathrm{mM}$ of desired NAC and the culture supernatant was analyzed after $24 \mathrm{~h}$ of growth for the presence of nitrite or ammonia, respectively. The results obtained showed that 2,4dinitrophenol, 2,5-dinitrophenol, and 2,6-dintirophenol were transformed oxidatively as determined by the release of nitrite molecule in the culture supernatant whereas $o$ dinitrobenzene, $m$-dinitrobenzene, $m$-nitrophenol, and 3,5-dinitrobenzoic acid were degraded reductively as determined by the release of ammonia in the medium (data not shown). However, $o$-nitrophenol, $p$-nitroaniline, and 2,3-dinitrotoulene were not transformed at all by the organism as also indicated by the non-detection of either nitrite or ammonia in the culture medium. To study the transformation products thin layer chromatography (TLC) and gas chromatography (GC) studies were performed using above-mentioned NACs. The TLC studies showed the presence of some metabolites in the culture

Table 1

Biotransformation of different NACs by Ralstonia sp. SJ98

\begin{tabular}{|c|c|c|c|c|}
\hline & \multicolumn{2}{|c|}{ GC Rt of substrate and metabolite(s) (min) } & \multicolumn{2}{|c|}{ HPLC Rt of substrate and metabolite(s) (min) } \\
\hline & Rt of substrate & Rt of metabolite(s) & Rt of substrate & Rt of metabolite(s) \\
\hline$o$-Dinitrobenzene & 2.743 & $2.242,2.631$ & 2.486 & $1.569,2.119,2.375$ \\
\hline$m$-Nitrophenol & 2.677 & N.D. & 2.164 & 1.641 \\
\hline 2,4-Dinitrophenol & 2.822 & 3.741 & 1.550 & 2.175 \\
\hline 2,5-Dinitrophenol & 2.730 & 2.931 & 1.664 & 2.046 \\
\hline
\end{tabular}

N.D., not detected. 
supernatants only in those cases where either nitrite or ammonia was detected (data not shown). The GC studies were then carried out on the extracted samples and these results are shown in Table 1. As indicated in this table, $o$ dinitrobenzene, 2,4-dinitrophenol, 2,5-dinitrophenol, 2,6-dinitrophenol, and 3,5-dinitrobenzoic acid showed the presence of metabolites based on their retention time (Rt). However, no metabolites could be detected in the case of $m$-dinitrobenzene and $m$-nitrophenol. To further confirm the presence of metabolites/transformation products of the degradative pathways, HPLC studies were also carried out on samples as described above. This also indicated the presence of different metabolites in degradative pathways based on their Rt (Table 1). However, we did not make attempts to identify these metabolites at this stage since the primary objective of this work
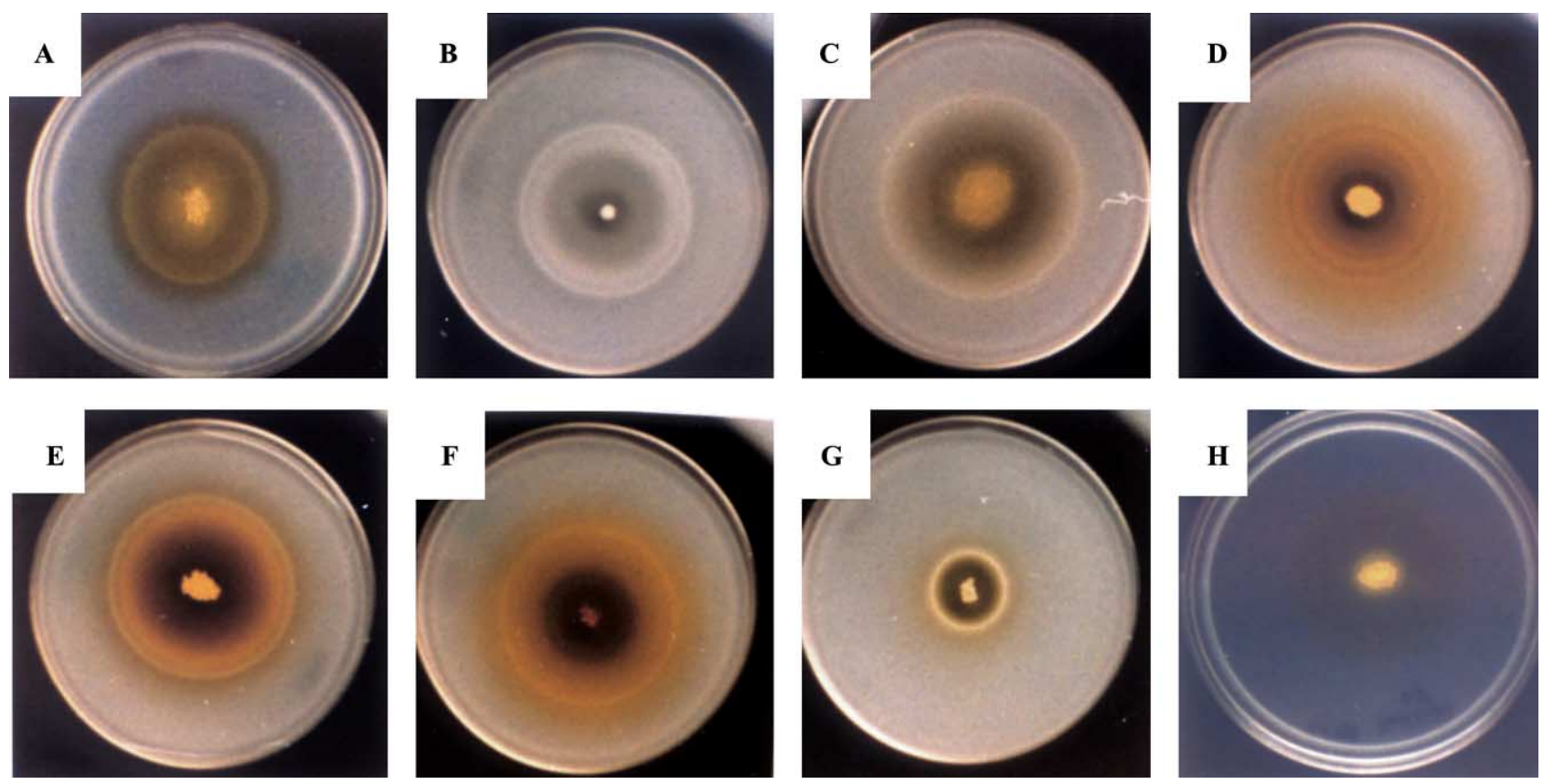

Fig. 1. Chemotaxis response of Ralstonia sp. SJ98 in drop assay. (A) $o$-Dinitrobenzene, (B) $m$-dinitrobenzene, (C) $m$-nitrophenol, (D) 2,4-dinitrophenol, (E) 2,5-dinitrophenol, (F) 2,6-dinitrophenol, (G) 3,5-dinitrobenzoate, and (H) o-nitrophenol (negative control).
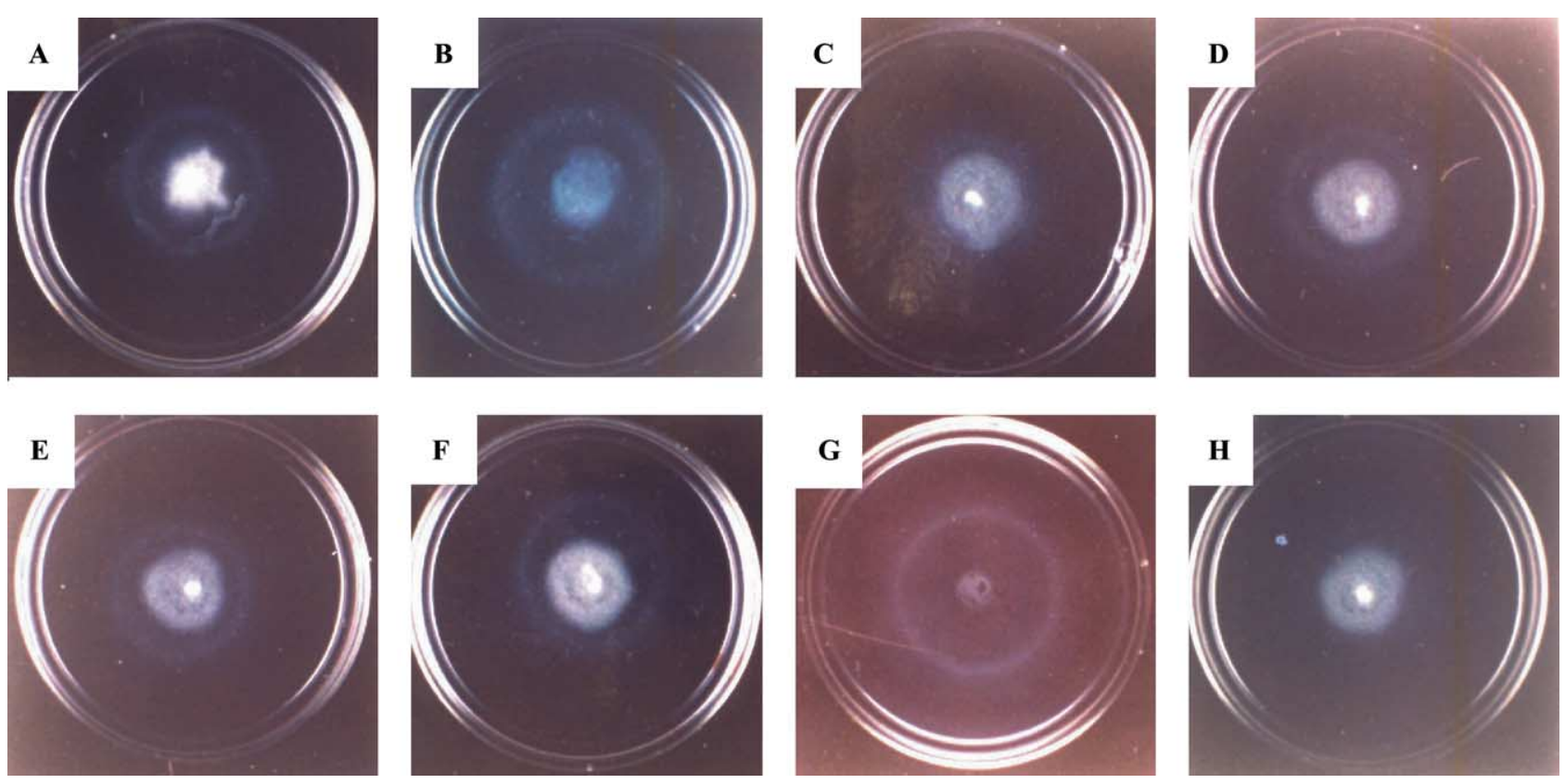

Fig. 2. Chemotaxis response of Ralstonia sp. SJ98 in swarm plate assay. (A) $o$-Dinitrobenzene, (B) $m$-dinitrobenzene, (C) $m$-nitrophenol, (D) 2,4dinitrophenol, (E) 2,5-dinitrophenol, (F) 2,6-dinitrophenol, (G) 3,5-dinitrobenzoate, and (H) o-nitrophenol (negative control). 
was to study the phenomenon of chemotaxis in relation to the transformation of the above-mentioned NACs. The identification of these metabolic pathways is the subject of further investigation.

We tested chemotaxis of strain SJ98 toward abovementioned NACs by drop assay, swarm plate assay, and capillary assay. The positive results of drop and swarm plate assays in the form of migrating rings of the bacteria are shown in Figs. 1 and 2, respectively. These results have shown that strain SJ98 is chemotactic toward only those seven NACs which are transformed by the strain SJ98 in the presence of succinate as a rapid metabolizable carbon and energy source (o-dinitrobenzene, $m$-dinitrobenzene, $m$-nitrophenol, 2,4-dinitrophenol, 2,5-dinitrophenol, 2,6-dintirophenol, and 3,5-dinitrobenzoic acid). The chemotactic behavior of the strain SJ98 was also quantitatively analyzed essentially by capillary assay. It was observed that strain SJ98 was chemotactic toward different co-metabolizable NACs with a chemotaxis index (C.I.) ranging from 3 to 37 at their optimum concentration (Table 2). Among seven NACs tested the organism was found to be highly chemotactic toward o-dinitrobenzene (C.I. of 37). However, strain SJ98 did not show any chemotaxis towards some other NACs, namely $o$-nitrophenol, $p$-nit- roaniline, and 2,3-dinitrotoulene; as indicated above, these NACs are also not transformed by strain SJ98. Taken together, the results obtained clearly indicated a correlation between chemotaxis of strain SJ98 toward NACs and their degradation/transformation.

There are three molecular mechanisms known in the literature for chemotaxis in bacteria [16]. Transmembrane signaling by bacterial chemoreceptors is the best studied mechanism where ligand binding to the external domain of the membrane spanning receptor generates a transmembrane signal that modulates kinase activity inside the cell, resulting in chemotaxis $[17,18]$. This signaling mechanism of chemotaxis is totally independent of metabolism of the effector molecule, and non-metabolizable structural analogues of the substrate can also elicit a chemotactic response $[16,18,19]$. This mechanism may not be operative in strain SJ98 toward NACs because the organism is attracted toward only those NACs that either serve as a source of carbon and energy to the cells or those that are co-metabolized in the presence of succinate as an alternate carbon source.

The second molecular mechanism known for chemotaxis depends on changes in the cellular energy levels resulting from the complete metabolism of chemoeffec-

Table 2

Chemotaxis response of Ralstonia sp. SJ98 in capillary assay toward different NACs

\begin{tabular}{|c|c|c|c|}
\hline Chemical & Structure & Optimum concentration $(\mu \mathrm{M})^{\mathrm{a}}$ & Chemotaxis index (C.I. $)^{\mathrm{b}}$ \\
\hline$o$-Dinitrobenzene & & 200 & 37 \\
\hline$m$-Dinitrobenzene & & 200 & 4 \\
\hline 2,4-Dinitrophenol & & 200 & 3 \\
\hline 2,6-Dinitrophenol & & 200 & 8 \\
\hline 3,5-Dinitrobenzoate & & 200 & 19 \\
\hline Aspartate ${ }^{\mathrm{c}}$ & & 200 & 60 \\
\hline
\end{tabular}

\footnotetext{
${ }^{\mathrm{a}}$ The optimum concentration reported here is the best of the three concentrations used, i.e., 10, 20, and $200 \mu \mathrm{M}$ to achieve the maximum C.I.

${ }^{\mathrm{b}}$ The chemotaxis assay was performed in triplicate and the results given here are means of these values. The variations are within $10 \%$.

${ }^{\mathrm{c}}$ Aspartate was used as positive control in these experiments.
} 
tor molecule $[16,20]$. This chemotaxis response is known as metabolism-dependent chemotaxis and it is reported in several microorganisms including Escherichia coli [21,22], Rhodobacter sphaeroides [23,24], and Azospirillum brasilense [25]. In $R$. sphaeroides, metabolism is a prerequisite for chemotactic movement toward sugars and ammonia [23,24]. Similarly, proline and glycerol are also reported to elicit chemotactic response of $E$. coli only after their metabolism [21,22]. The co-metabolism of NACs may be partially responsible for chemotactic response in strain SJ98; however, whether this can generate a detectable change in energy flux of the cell needed for metabolism-dependent chemotaxis is yet to be ascertained.

The third mechanism of chemotaxis argues that the signal for chemotaxis is generated along with the transport of the molecule to the cell [16]. For example, chemotaxis of E. coli and Bacillus subtilis toward sugars [26] and carbohydrates [27], respectively, is coupled to the transport of the chemoattractant. In Pseudomonas putida, pcaK encodes a non-essential transporter for 4hydroxybenzoate that is required for chemotaxis towards this compound [28]. Similarly, $t f d K$, a permease that allows Ralstonia eutropha JMP123 to take up very low concentrations of 2,4-dichlorophenoxyacetate (2,4D) but is not essential for its entry into the cells, is required for chemotaxis to 2,4-D [29]. It is not understood how PcaK or TfdK plays a role in chemotaxis but transport through these permeases could be generating a signal for chemotaxis toward these environmental pollutants. Chemotaxis of strain SJ98 toward co-metabolizable NACs could also be coupled to transport. We argue that either transport, transformation, and signal generation for chemotaxis in strain SJ98 occur as a single event, or, transformation and signal generation occur as a single event by intracellular enzymes, after transport. We are currently identifying the molecular mechanisms operating behind chemotaxis of strain SJ98 to metabolizable/co-metabolizable NACs.

\section{Acknowledgments}

We are grateful to Dr. Amit Ghosh, Director, for his encouragement. This work, in part, was supported by the Indo-Swiss Collaboration in Biotechnology (ISCB). This is IMTECH Communication No. 020/2002.

\section{References}

[1] S.K. Samanta, O.V. Singh, R.K. Jain, Polycyclic aromatic hydrocarbons: environmental pollution and bioremediation, Trends Biotechnol. 20 (2002) 243-248.

[2] K.N. Timmis, D.H. Pieper, Bacteria designed for bioremediation, Trends Biotechnol. 17 (1999) 201-204.

[3] S. Balajee, A. Mahadevan, Potential role of engineered microbes to de-toxify chemical pollutants, MIRCEN J. 5 (1989) 119-134.
[4] S.E. Hebes, I.R. Rowsell, Microbial degradation of polycyclic aromatic hydrocarbons in pristine and petroleum-contaminated sediments, Appl. Environ. Microbiol. 35 (1986) 306316.

[5] S.K. Samanta, B. Bhushan, A. Chauhan, R.K. Jain, Chemotaxis of a Ralstonia sp. SJ98 toward different nitroaromatic compounds and their degradation, Biochem. Biophys. Res. Commun. 269 (2000) 117-123.

[6] B. Bhushan, S.K. Samanta, A. Chauhan, A.K. Chakraborti, R.K. Jain, Chemotaxis and degradation of 3-methyl-4-nitrophenol by Ralstonia sp. SJ98, Biochem. Biophys. Res. Commun. 275 (2000) 129-133.

[7] S.K. Samanta, R.K. Jain, Evidence for plasmid-mediated chemotaxis of Pseudomonas putida towards naphthalene and salicylate, Can. J. Microbiol. 28 (2000) 683-690.

[8] A.C. Grimm, C.S. Harwood, Chemotaxis of Pseudomonas spp. to the polyaromatic hydrocarbon, naphthalene, Appl. Environ. Microbiol. 63 (1997) 4111-4115.

[9] R.E. Parales, J.L. Ditty, C.S. Harwood, Toluene-degrading bacteria are chemotactic towards the environmental pollutants benzene, toluene, and trichloroethylene, Appl. Environ. Microbiol. 66 (2000) 4098-4104.

[10] R.E. Parales, C.S. Harwood, Bacterial chemotaxis to pollutants and plant-derived aromatic molecules, Curr. Opin. Microbiol. 5 (2002) 266-273.

[11] J.C. Spain, Biodegradation of nitroaromatic compounds, Annu. Rev. Microbiol. 49 (1995) 523-555.

[12] L.H. Keith, W.A. Telliard, Priority pollutants I. A prospective view, Environ. Sci. Technol. 13 (1976) 416-423.

[13] J.C. Spain, J.B. Hughes, H.J. Knackmuss, Biodegradation of Nitroaromatic Compounds and Explosives, Lewis Publishers, Washington, DC, 2000.

[14] M. Rani, R.C. Sobti, R.K. Jain, Plasmid-mediated degradation of $o$-phthalate and salicylate by a Moraxella sp., Biochem. Biophys. Res. Commun. 220 (1996) 377-381.

[15] D. Prakash, A. Chauhan, R.K. Jain, Plasmid-encoded degradation of $p$-nitrophenol by Pseudomonas cepacia, Biochem. Biophys. Res. Commun. 224 (1996) 375-381.

[16] G. Alexander, I.B. Zhulin, More than one way to sense chemicals, J. Bacteriol. 183 (2001) 4681-4686.

[17] A. Bren, M. Eisenbach, How signals are heard during bacterial chemotaxis: protein-protein interactions in sensory signal propagation, J. Bacteriol. 182 (2000) 6865-6873.

[18] J.J. Falke, G.L. Hazelbauer, Transmembrane signaling in bacterial chemoreceptors, Trends Biochem. Sci. 26 (2001) 257 265.

[19] J. Adler, Chemoreceptors in bacteria, Science 166 (1969) 1588 1597.

[20] B.L. Taylor, I.B. Zhulin, In search of higher energy: metabolismdependent behavior in bacteria, Mol. Microbiol. 28 (1998) 683690.

[21] M. Clancy, K.A. Mandill, J.M. Wood, Genetic and biochemical requirements for chemotaxis to L-proline in Escherichia coli, J. Bacteriol. 146 (1981) 902-906.

[22] I.B. Zhulin, E.H. Rowsell, M.S. Johnson, B.L. Taylor, Glycerol elicits energy taxis of Escherichia coli and Salmonella typhimurium, J. Bacteriol. 179 (1997) 3196-3201.

[23] Y. Jezoire-Sassoon, P.A. Hamblin, C.A. Bootle-Wilbraham, P.S. Poole, J.P. Armitage, Metabolism is required for chemotaxis to sugars in Rhodobacter sphaeroides, Microbiology 144 (1998) 229-239.

[24] P.S. Poole, J.P. Armitage, Role of metabolism in the chemotactic response of Rhodobacter sphaeroides to ammonia, J. Bacteriol. 171 (1989) 2900-2902.

[25] G. Alexander, S.E. Greer, I.B. Zhulin, Energy taxis is a dominant behavior in Azospirillum brasilense, J. Bacteriol. 182 (2000) 60426048 . 
[26] J. Adler, W. Epistein, Phosphotransferase-system enzymes as chemoreceptors for certain sugars in Escherichia coli chemotaxis, Proc. Natl. Acad. Sci. USA 71 (1974) 2895-2899.

[27] L.F. Garrity, S.L. Merrill, J. Reizer, M.H. Saier Jr., G.W. Ordal, Unique regulation of carbohydrate chemotaxis in Bacillus subtilis by the phosphoenolpyruvate-dependent phosphotransferase system and the methyl accepting chemotaxis protein McpC, J. Bacteriol. 180 (1998) 4475-4480.
[28] C.S. Harwood, N.N. Nichols, M.-K. Kim, J.L. Ditty, R.E Parales, Identification of a pcaRKF gene cluster from Pseudomonas putida: involvement in chemotaxis, biodegradation, and transport of 4-hydroxybenzoate, J. Bacteriol. 176 (1994) 64796488.

[29] A.C. Hawkins, C.S. Harwood, Chemotaxis of Ralstonia eutropha JMP123(pJP4) to the herbicide 2,4-dichlorophenoxyacetate, Appl. Environ. Microbiol. 68 (2002) 968-972. 